\title{
Symptomatic sarcoidosis of skeletal muscle
}

\author{
A. C. DOUglas, J. G. MACleod, AND J. D. MATtheWS \\ From the Department of Respiratory Diseases, University of Edinburgh, the University \\ Department of Medicine, Western General Hospital, and the Royal Infirmary, Edinburgh
}

SUMMARY Two patients with chronic sarcoid myopathy are described. Both were middle-aged females and both showed the features of pseudohypertrophy. In other aspects they contrasted markedly. In one (A.R.) the sheer volume of granuloma and its effect on muscle fibres was sufficient to explain the muscle weakness and electromyography confirmed a pure myopathy. In the other (J.W.) the muscle granuloma was sparsely distributed and an associated neuropathy contributed importantly to the disability. There was no clinical evidence of sarcoidosis of other organs in one (A.R.) but necropsy showed multisystem involvement. There was clinical and radiographic evidence of widespread sarcoidosis in the other (J.W.). Both patients showed an initial dramatic response to prednisolone. The reported literature of symptomatic muscle sarcoidosis is reviewed briefly.

While asymptomatic involvement of skeletal muscle in sarcoidosis is sufficiently common to make muscle biopsy one of the accepted methods of tissue diagnosis (Myers et al., 1952; Phillips and Phillips, 1956; Wallace et al., 1958), symptomatic involvement is rare and only 44 cases have been recorded. There are three main clinical groups:

1. Muscle pain and tenderness due to acute myositis may occur in the early stages of sarcoidosis (Silverstein and Siltzbach, 1969). The symptoms are rapidly controlled by corticosteroid therapy and there is no relapse on withdrawal provided treatment has been sufficiently prolonged (about six months as a rule).

2. Palpable muscle nodules, the first form of muscle sarcoidosis to be reported (Licharew, 1908) but the least common, may present with pain and stiffness or muscle cramps (Bates and Walsh, 1948; Eguro and Yabuki, 1966). Muscle weakness may not be a feature during the active phase of this form of the disease but can be conspicuous later if contractures occur (Snorrason, 1947). It is possible that some of the cases recorded were, in fact, examples of skin and subcutaneous tissue involvement along with asymptomatic muscle involvement (Silverstein et al., 1969).

3. Symptomatic muscle sarcoidosis may pre- sent as a chronic myopathy, with bilateral weak ness and wasting of the proximal muscles of the 을 extremities and of the trunk and neck muscles the clinical picture resembling that of muscula $\mathbb{D}$ dystrophy (Devic et al., 1955; Ammitzböllo $₫$ 1956; McConkey, 1958; Erbslöh and Dietele 1959; Harvey, 1959; Kryger and Rønnov-Jessen⿳亠口冋 1959; Brun, 1961; Crompton and MacDermot, $\vec{\varphi}$ 1961; Hinterbuchner and Hinterbuchner, 1964; Hofstetter, 1967; Talbot, 1967). Muscle pain may precede the weakness and atrophy. The similarity to muscular dystrophy is increased when pseudohypertrophic features occur.

In over 500 patients with sarcoidosis seen in the Edinburgh area over the past 20 years there have been only two cases of symptomatic sarcoidosis of skeletal muscle. Both were examples of chronic myopathy and both showed the features of pseudohypertrophy.

\section{CASE 1}

A.R., a 59 year old married woman with no medical history of note, became suddenly unwell in March 1964 with general malaise, fever, and oliguria. Two weeks later the ankles became oedematous and the leg muscles became painful, stiff, and weak. Over the next two months the urine volume returned to normal but the peripheral oedema persisted and worsened, and increased muscular weakness made walking difficult. Despite the oedema there was 034 


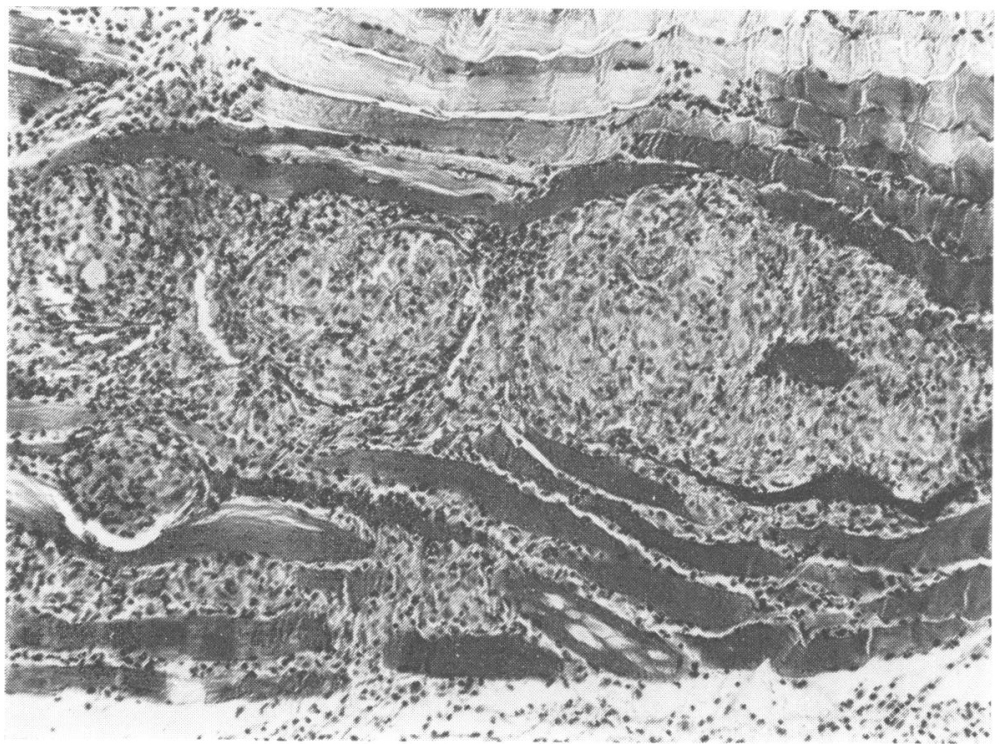

FIG. 1. Patient A.R. Muscle biopsy showing marked granuloma formation between muscle fibres. $H$ and $E, \times 110$.

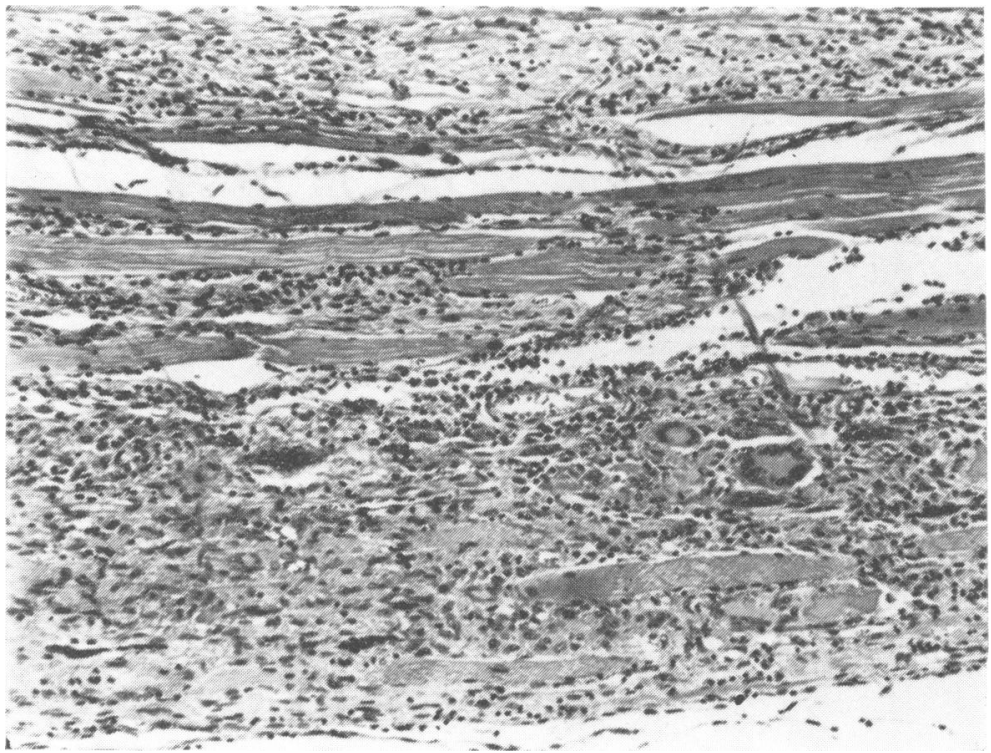

FIG. 2. Patient A.R. Skeletal muscle at necropsy showing extensive granulomatous change and atrophy and degeneration of muscle fibres. $H$ and $E, \times 120$.

marked loss of weight amounting to $12.7 \mathrm{~kg}(28 \mathrm{lb})$ over four months. On admission to hospital in August 1964 the dominant clinical finding was marked pitting oedema of both legs. The leg muscles were bilaterally weak and tendon reflexes were absent. All other muscles were clinically normal and there were no sensory changes. There was proteinuria, microscopic haematuria, a raised blood urea, impaired creatinine clearance and a mild normochromic, normocytic anaemia. A clinical diagnosis of nephritis was made and renal biopsy showed features compatible with resolving glomerulonephritis. On symptomatic treatment the clinical state improved and after six weeks the patient was discharged with no overt oedema, a normal blood urea, and only a trace of albumin in the urine. The leg 


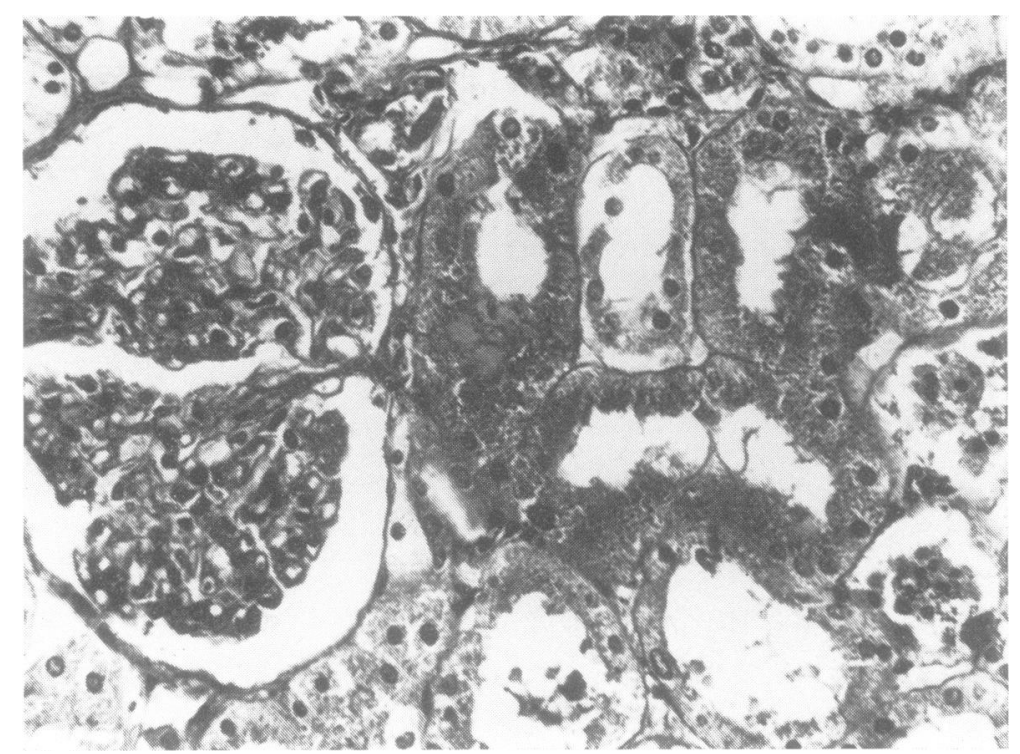

FIG. 3. Patient A.R. Necropsy specimen of kidney showing hyaline droplets in convoluted tubules. $H$ and $E, \times 300$.

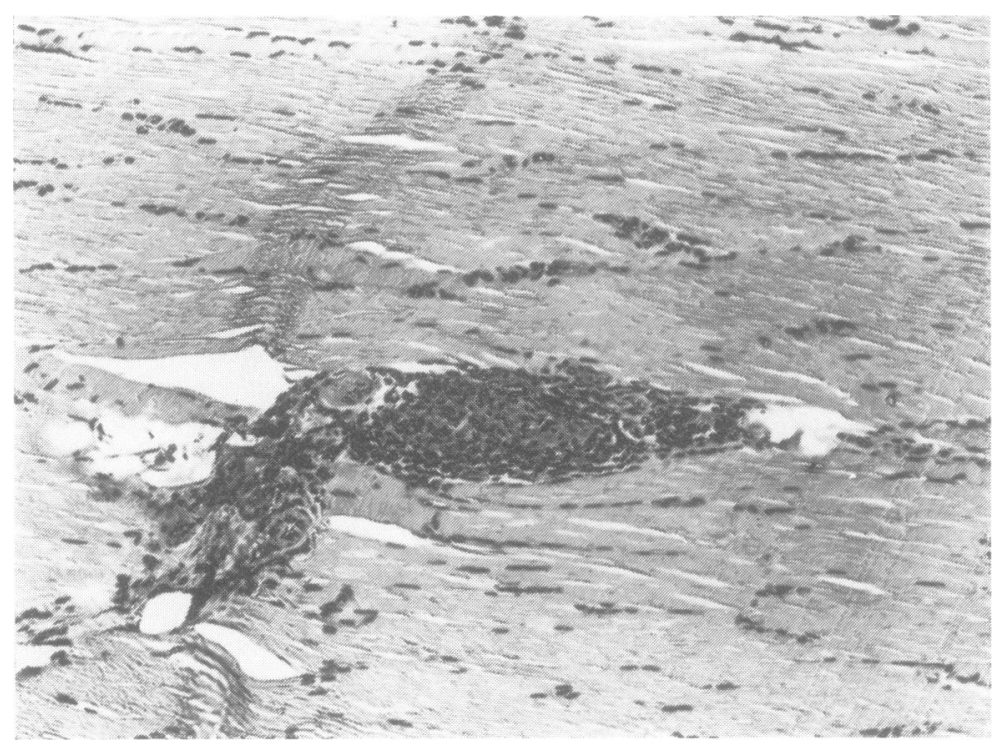

FIG. 4. Patient J.W. Muscle biopsy showing scanty sarcoid granulomata. $H$ and $E, \times 120$.

weakness had markedly improved. For some weeks she was relatively well until she again noticed swelling of the ankles, and developed pain, weakness, and stiffness of the leg muscles particularly affecting the anterior thighs and the calves, which became swollen and tender over a period of about two weeks. On readmission to hospital in November 1964 there was marked swelling and weakness of the leg muscles on both sides, the appearances resembling pseudo- hypertrophic muscular dystrophy. Electromyography (EMG) of the left quadriceps, left anterior tibial, left calf, and left deltoid muscles showed that the majority of motor units were 'broken up' and myopathic in appearance. The EMG features were consistent with a diagnosis of myositis with a wider distribution than the clinical findings suggested. Biopsy of the quadriceps muscle showed exuberant granuloma formation of sarcoid type distending the muscle and widely 
separating the muscle fibres which, otherwise, were histologically normal (Fig. 1). No other organ involvement was demonstrated on clinical and radiographic examination. The Mantoux test was negative to $1: 100 \mathrm{O}$.T. The serum protein levels were normal.

Treatment with prednisolone was begun in a dosage of $20 \mathrm{mg}$ daily. This had a dramatic effect and within a week the patient, who had previously had difficulty in standing, was able to walk short distances. By the end of a month she had virtually regained normal muscular power. The muscle pain subsided rapidly and after two weeks the pseudohypertrophy was no longer evident. Over the next six months improvement was maintained on regular treatment with prednisolone in a dosage of $10 \mathrm{mg}$ daily, but in June 1965 there was slight but definite deterioration in muscular power after a reduction in the dosage of prednisolone to $7.5 \mathrm{mg}$ daily which was made because of severe persisting dyspepsia and ulcer-type pain occurring in association with a sliding hiatus hernia. The deterioration was rapidly controlled by increasing the dose of prednisolone, but by October epigastric pain and dyspepsia had again become so distressing that a further reduction in the dosage of prednisolone was attempted. This was followed by severe relapse of muscle symptoms and it was necessary to readmit the patient to hospital in December 1965. The leg muscles were painful, weak, and swollen, the proximal muscles of the upper limbs were also very weak, and there was now persisting fever and a raised serum globulin. Despite increase in the dosage of prednisolone to $20 \mathrm{mg}$ daily the patient became weaker. Dyspnoea developed, raising the possibility of involvement of the muscles of respiration. The cerebrospinal fluid (CSF) was examined at this time and was normal. The patient died suddenly in February 1966 with the clinical features of massive pulmonary embolism.

At necropsy pulmonary embolism was confirmed. The voluntary muscles (Fig. 2) and the liver were involved extensively by active sarcoidosis and sarcoid follicles were also demonstrated in the spleen, bones, tongue, and lymph nodes. The central nervous system was normal. The kidneys showed epithelial proliferation in a few of the glomeruli with thickening of the basement membranes consistent with previous glomerulonephritis. The most conspicuous renal abnormality, however, was the presence of hyaline droplets in the cytoplasm of the convoluted tubules (Fig. 3). These were eosinophilic, stained brightly red with Picro-Mallory and yellow with Van Gieson, were PAS negative, and did not show metachromia with Cresyl-Echt Violet. The droplets were thought to be protein in the process of transport through the tubular cells and it seemed possible that myoglobin may have contributed to their formation, since the breakdown of muscle fibres was so marked and widespread. In contrast with the initial histological appearances, the skeletal muscle fibres at necropsy were grossly abnormal and showed the features of atrophy and degeneration.

An association has been suggested between myocardial and skeletal muscle sarcoidosis (Powell. 1953) but in this patient there was no necropsy evidence of myocardial involvement.

\section{CASE 2}

J.W., a 56 year old unmarried woman, presented in July 1971 with a three month history of swelling and tenderness of the legs and swelling of the abdomen. There was progressive loss of power in the legs associated with ataxia so that she was 'uncertain where her legs were going'. She had a long-standing sensitivity to cold in her fingers but for about two months she also had numbness and tingling of the fingers and toes, and for one month stiffness and swelling of the hands, particularly of the dorsum of the first and second fingers, dryness of the mouth, and swelling of both parotid glands.

On examination both legs were swollen and there was ankle and sacral oedema. In both lower limbs there was a flaccid weakness with absent tendon reflexes, impaired vibration and position sense, and there was loss of fine touch sensation on the tips of the fingers and toes. The skin of the fingers was red, thickened, and tight, causing limitation of flexion. Radiographs of the hands showed normal bones. Her cheeks had a high colour and there was firm, painless enlargement of both parotid glands. The abdominal muscles were very lax and explained the abdominal swelling. The upper limb muscles were clinically normal. There was no evidence of ocular involvement. The jugular venous pressure was raised. The peripheral blood was normal and the erythrocyte sedimentation rate (ESR) was only $7 \mathrm{~mm}$ per hour. The Mantoux test was negative to $1: 100$ O.T. The CSF showed 19 lymphocytes per $\mathrm{cu}$. $\mathrm{mm}$ and a protein of $128 \mathrm{mg} / 100 \mathrm{ml}$. The electroencephalogram (EEG) was normal. Electromyography of the right calf, right quadriceps, right forearm extensors, right biceps, and right and left deltoid muscles showed a mixed picture of neuromyopathy with a predominance of myopathic changes. Although the patient did not complain of dyspnoea, tests of ventilatory function on 13 August 1971 showed a restrictive ventilatory abnormality with a Forced Vital Capacity (FVC) of 2,240 ml. and a Forced Expiratory Volume in one second $\left(\mathrm{FEV}_{1}\right)$ of $1,760 \mathrm{ml}$. as opposed to the predicted values of $3,100 \mathrm{ml}$. and $2,450 \mathrm{ml}$. respectively. The transfer 
factor for carbon monoxide (TCO) was slightly reduced. The chest radiograph revealed bilateral hilar adenopathy. Muscle biopsy from gastrocnemius, quadriceps, and rectus abdominis muscles showed diffuse granulomatous lesions compatible with sarcoidosis. The lesions, which were centred on small blood vessels of capillary calibre between bundles of apparently normal muscle fibres, were scanty and, histologically, the extent of the muscle involvement was not commensurate with the severity of the muscular weakness (Fig. 4). Since there was no muscle atrophy, it was evident that the associated neuropathy contributed importantly. An electrocardiogram (ECG) showed sinus rhythm and evidence of left ventricular hypertrophy. There was radiological enlargement of the heart, the $C T$ ratio being 170/270. Aspartate aminotransferase and hydroxybutyrate dehydrogenase levels were normal in the blood on three occasions. A barium swallow showed normal peristalsis. The serum electrolytes,

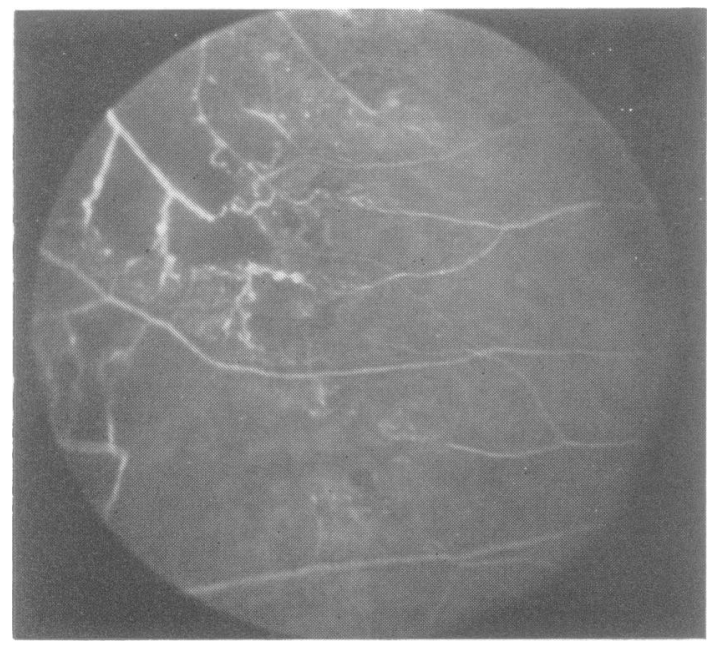

FIG. 5. Patient J.W. Fluorescein retinogram showing features of retinal vasculitis.

bilirubin, alanine and aminotransferase, and alkaline phosphatase were normal. The serum creatinine was $96 \mathrm{mg} / 100 \mathrm{ml}$., calcium $9.7 \mathrm{mg} / 100 \mathrm{ml}$, and phosphate $3.2 \mathrm{mg} / 100 \mathrm{ml}$. The electrophoretic pattern of plasma proteins was normal and the antinuclear factor was present in the blood on one occasion.

During the course of investigations the bilateral parotid swelling subsided spontaneously and the legs became less swollen and painful, although there was no improvement in the muscular weakness which was most marked in the thighs. Treatment with frusemide resulted in a marked diuresis and cleared the peripheral oedema. Corticosteroid therapy was begun with prednisolone in a dosage of $20 \mathrm{mg}$ daily, which resulted in very satisfactory clinical improvement. The residual swelling of the legs subsided slowly over four weeks and the leg and abdominal muscles steadily regained their power. As tone returned to the recti the protuberance of the abdomen subsided. The subcutaneous tissue swellings of the fingers slowly improved. On discharge from hospital in October 1971 the patient was able to walk freely.

At review in late November muscular power in both legs had completely returned to normal and the abdominal muscles had recovered normal power. The lower limb tendon reflexes were, however, still absent. It was interesting that ventilatory function was normal $\left(\mathrm{FEV}_{1} 2,400 \mathrm{ml}\right.$., FVC 3,100 ml.), suggesting that there may have been initial involvement of respiratory muscles which improved with treatment.

The muscle sarcoidosis continued to be well controlled on maintenance treatment with prednisolone now reduced to $17.5 \mathrm{mg}$ daily, but in February 1972 the patient began to complain of impairment of vision in the right eye and investigation showed vitreous opacities and numerous peripheral super ficial retinal haemorrhages attributed to the develop ment of vasculitis, the appearances being very simila? to those of Eales's disease (Fig. 5). The left eye showe 6 . only a few haemorrhagic spots. It seemed mos probable that the retinal changes were an expression of the sarcoid process which was not being completely suppressed by prednisolone. On this account the dosage was increased temporarily to $30 \mathrm{mg}$, with subsequent improvement.

At one stage during follow-up the pulse was noted to be grossly irregular in time and force and a clinical diagnosis of atrial fibrillation was made, but by the time an ECG was obtained the rhythm had reverted to sinus. The patient continues to require large doses of diuretics to control peripheral oedema which is fairly certainly of cardiac origin. Whether or not it is aetiologically related to sarcoid involvement of the heart muscle is a matter for speculation. All other sarcoid features are under excellent control on treatment with prednisolone $20 \mathrm{mg}$ daily.

\section{DISCUSSION}

The pathogenesis of symptomatic muscle sarcoidosis is obscure and why it is that the presence of granuloma should be associated with symptoms in some cases but is wholly asymptomatic 
in the vast majority is not known. Muscle lesions can occur in the course of systemic sarcoidosis long before a myopathic picture develops (Coers and Carbone, 1966), but the reason for the disease process becoming dominant in skeletal muscles remains a mystery. It has been suggested that previous non-sarcoid muscle disease may predispose but this is not borne out by most case histories. The predilection for menopausal women has led to speculation about an endocrine factor but there is no proof of this (Hinterbuchner et al., 1964).

The extent of granuloma formation in the myopathic forms of muscle sarcoidosis can vary greatly and this is well demonstrated in the two patients reported here. In the first (A.R.) the volume of granuloma alone and its effect on muscle fibres was sufficient to explain the muscular weakness, and the absence of clinical or EMG evidence of neuropathy indicated a pure myopathy. The CSF was normal. In the second (J.W.) the granuloma was sparsely distributed and the muscle fibres showed no histological abnormality but there was definite clinical and EMG evidence of associated neuropathy. There was also CSF abnormality indicating involvement of the central nervous system. Nevertheless in these two cases the clinical picture was remarkably similar and the evidence of pseudohypertrophy was equally pronounced. It is unusual for the granulomatous process to be so marked as it was in patient A.R.; in general, disability in symptomatic muscle sarcoidosis depends more on muscle atrophy and nervous system involvement than on the extent of granulomatous change.

In patient J.W. the evidence of cardiac abnormality developing coincident with florid widespread sarcoid phenomena suggests a common aetiology and supports Powell's contention that in chronic sarcoid myopathy cardiac involvement frequently coexists (Powell, 1953).

Had there been no necropsy, patient A.R. might have been added to the 22 cases reported in the literature as isolated sarcoidosis of skeletal muscle (Devic et al., 1955; Ammitzböll, 1956; Erbslöh and Dietel, 1959; Harvey, 1959; Kryger and Rønnov-Jessen, 1959; Crompton and MacDermot, 1961; Hinterbuchner and Hinterbuchner, 1964; Coërs and Carbone, 1966; Talbot, 1967). Isolated muscle sarcoidosis has, however, never been confirmed by necropsy and it is doubtful if such an entity exists.

The place of muscle biopsy in sarcoidosis is now limited. Although muscle biopsy may give a histological diagnosis in 50 to $80 \%$ of patients with early sarcoidosis (Myers et al., 1952; Maurice, 1955; Phillips and Phillips, 1956; Wallace et al., 1958) the procedure has largely been made superfluous by the Kveim test and can now rarely be justified. Muscle biopsy has not been used routinely in the diagnosis of chronic sarcoidosis and its value in this context is not known. Muscle biopsy, however, plays a definitive role in symptomatic muscle sarcoidosis. Any non-familial muscular dystrophy should be considered for muscle biopsy (Rothfeld and Folk, 1962). Other conditions which may on occasion be simulated by chronic sarcoid polymyositis are myasthenia gravis (Devic et al., 1955), dermatomyositis (Weinberger, 1933), progressive muscular atrophy (deMorsier et al., 1954), and, when there are associated joint symptoms, Still's disease (Castellanos and Galan, 1946).

Recorded evidence of the value of corticosteroid therapy in chronic sarcoid myopathy is equivocal. Fourteen patients, including the two reported here, have benefited from corticosteroids and nine have not. Corticosteroids must always be given a trial, and it is important that the dosage is adequate and that treatment is sufficiently prolonged. Both patients in this report improved remarkably on treatment with prednisolone. In one the muscle symptoms have been kept completely in check for more than a year after the onset. To what extent the outcome in patient A.R. would have been different if it had been possible to maintain a high level of dosage of prednisolone throughout is debatable. Obviously dosages lower than $10 \mathrm{mg}$ per day cannot be relied upon as maintenance therapy in those patients in whom initial improvement has been achieved with higher dosage. Treatment may require to be continued indefinitely.

\section{CONCLUSION}

Sarcoid myopathy is a chronic form of sarcoidosis which classically presents in middle age or later. Usually there is diffuse symmetrical polymyositis with muscle atrophy, but occasionally 
there are palpable nodules or pseudohypertrophy or, in long-standing cases, fibrosis and induration or contractures. Proximal muscles are most frequently involved clinically but, histologically, there is often widespread muscle involvement. The condition may simulate progressive muscular atrophy or muscular dystrophy. Neuropathy may coexist and electromyography is useful in determining the extent to which it is contributing to the symptomatology. There may or may not be evidence of sarcoidosis in other systems when symptomatic muscle sarcoidosis becomes evident, but in those cases in which necropsies have been performed multisystem involvement has always been demonstrated. Muscle biopsy plays a definitive role in diagnosis and should always be considered in neuromuscular disorders which present with unusual features or which follow an unusual course. In chronic sarcoid myopathy the muscle fibres are not invaded by granuloma but there may be pressure atrophy or degeneration with fibrosis. The effect of corticosteroid therapy is variable, probably depending on the duration of the process and possibly on the extent of associated neurological involvement. A trial of prednisolone should always be given, and if a response is demonstrated it is vital that the maintenance dosage is kept at an adequate level, at least $10 \mathrm{mg}$ per day.

We wish to thank Dr. N. McLean and Dr. C. P. Mayers for the pathological reports and Dr. I. D. Sanderson and Dr. I. R. Williams for the EMG reports.

\section{REFERENCES}

Ammitzbøll, F. (1956). A case of Boeck's sarcoid with isolated localization in the musculature. Acta Rheumatologica Scandinavica, 2, 3-10.

Bates, G. S., and Walsh, J. M. (1948). Boeck's sarcoid: observations on seven patients, one autopsy. Annals of Internal Medicine, 29, 306-317.

Brun, A. (1961). Chronic polymyositis on the basis of sarcoidosis. Acta Psychiatrica et Neurologica Scandinavica, 36, 515-523.

Castellanos, A., and Galan, E. (1946). Sarcoidosis (BesnierBoeck-Schaumann's disease): report of a case in a child simulating Still's disease. American Journal of Diseases of Children, 71, 513-529.
Coërs, C., and Carbone, F. (1966). La myopathie granulomateuse. Acta Neurologica et Psychiatrica Belgica, 66, 353381.

Crompton, M. R., and MacDermot, V. (1961). Sarcoidosis associated with progressive muscular wasting and weakness. Brain, 84, 62-74.

Morsier, G. de, Maurice, P., and Martin, F. (1954). BesnierBoeck diffus des muscles et lésions du système nerveux central. (Deux observations anatomocliniques). Acta Neurologica et Psychiatrica Belgica, 54, 34-51.

Devic, M., Masson, R., and Bonnefoy (1955). A propos d'une observation de myosite à nodules de Besnier-Boeck. Revue Neurologique, 92, 563-567.

Eguro, H., and Yabuki, M. (1966). Sarcoidosis of the skeletal muscle with tumor formation: a case report and review of the literature. Yokohama Medical Bulletin, 17, $75-85$.

Erbslöh, F., and Dietel, W. (1959). Über exogene Spätmyopathien. I. Die Polymyositis granulomatosa Boeck. Archiv für Psychiatrie und Nervenkrankheiten, 199, 215-234.

Harvey, J. C. (1959). A myopathy of Boeck's sarcoid. American Journal of Medicine, 26, 356-363.

Hinterbuchner, C. N., and Hinterbuchner, L. P. (1964). Myopathic syndrome in muscular sarcoidosis. Brain, 87, 355-366.

Hofstetter, J.-R. (1967). Forme amyotrophique grave de sarcoïdose. Traitement à la prednisone. In La Sarcoïdose, pp. 678-683. Masson: Paris.

Kryger, J., and Rønnov-Jessen, V. (1959). Myopathy in Boeck's sarcoid. Acta Rheumatologica Scandinavica, 5 314-322.

Licharew (1908). (Demonstration.) Dermatologisches zentral blatt, 11, 253-254.

McConkey, B. (1958). Muscular dystrophy in sarcoidosis Archives of Internal Medicine, 102, 443-446.

Maurice, P. A. (1955). La participation de la musculature la maladie de Besnier-Boeck-Schaumann. Étude anatomo clinique portant sur 13 cas. Helvetica Medica Acta, 22, 16 42.

Myers, G. B., Gottlieb, A. M., Mattman, P. E., Eckley G. M., and Chason, J. L. (1952). Joint and skeletal muscle manifestations in sarcoidosis. American Journal of Medicine, 12, 161-169.

Phillips, R. W., and Phillips, A. M. (1956). The diagnosis of Boeck's sarcoid by skeletal muscle biopsy. Archives of Internal Medicine, 98, 732-736.

Powell, L. W. Jr. (1953). Sarcoidosis of skeletal muscle. American Journal of Clinical Pathology, 23, 881-889.

Rothfeld, B., and Folk, E. E., III (1962). Sarcoid myopathy. Journal of the American Medical Association, 179, 903-905.

Silverstein, A., and Siltzbach, L. E. (1969). Muscle involvement in sarcoidosis. Archives of Neurology, 21, 235-241.

Snorrason, E. (1947). Myositis fibrosa progressiva-Lymphogranulomatosis benigna Boeck. Nordisk Medicin, 36, 24242425 .

Talbot, P. S. (1967). Sarcoid myopathy. British Medical Journal, 4, 465-466.

Wallace, S. L., Lattes, R., Malia, J. P., and Ragan, C. (1958). Muscle involvement in Boeck's sarcoid. Annals of Internal Medicine, 48, 497-511.

Weinberger, M. (1933). Über eine chronisch verlaufende Polymyositis mit Ausgang in progressive Muskelatrophie. Wiener Medizinische Wochenschrift, 83, 100-103. 